\title{
Dispersion Insensitive, High-Speed Optical Clock Recovery based on a Mode-Locked Laser Diode
}

\author{
P.J.Maguire, D.Reid and L.P.Barry \\ Research Institute for Networks and Communications Engineering, Dublin City University, Dublin 9, IRELAND. \\ Tel: +353 (0)1 700 6401, Fax: +353 (0) 17005508 maguirep@eeng.dcu.ie \\ A.O'Hare \\ School of Physics, Dublin Institute of Technology, Kevin St, Dublin 8, IRELAND. \\ S.Lobo, V.Roncin, M. Gay, L.Bramerie, and J.C.Simon \\ CNRS UMR FOTON 6082, PERSYST Platform, ENSSAT, Universite de Rennes 1,Lannion, FRANCE.
}

\begin{abstract}
An investigation into the effects of varying levels of chromatic dispersion on a ModeLocked Laser Diode optical clock recovery process is presented. Results demonstrate that this technique is invariant to input dispersion varying between $\pm 75 \mathrm{ps} / \mathrm{nm}$.

(C)2007 Optical Society of America

OCIS codes: (060.4510) Optical communications; (250.4745) Optical processing devices
\end{abstract}

\section{Introduction}

Future high capacity Optical Time Division Multiplexed (OTDM) networks operating at aggregate data rates greater than $100 \mathrm{~Gb} / \mathrm{s}$ will require optical clock recovery (OCR) to carry out various optical signal processing tasks [1]. A number of different OCR methods have been demonstrated [2,3], with the work reported here utilising a ModeLocked Laser Diode (MLLD) [4,5]. The advantage of this method is that when an RZ data stream is injected into the MLLD, it produces a sub-harmonic optical pulse train as an output [4]. Here a self-pulsating mode-locked quantum dot Fabry-Perot (ML-QD-FP) laser diode, provided by the III-V Lab in the framework of the French research program ROTOR, was used to recover a $42 \mathrm{GHz}$ optical clock from a $170 \mathrm{~Gb} / \mathrm{s}$ OTDM data signal [6]. Previous work $[7,8]$ involved the re-modulation of the recovered clock signal to carry out BER measurements to quantify the performance of the clock recovery process. Impairments such as timing jitter and optical noise would manifest themselves as an accumulated power penalty derived from the BER measurement. Here, the Frequency Resolved Optical Gating (FROG) [9] technique is used to directly characterise the performance of the OCR process to varying levels of input dispersion. The results presented confirm that this OCR technique produces a high quality clock signal that is independent of the level of dispersion that the data signal has experienced.

\section{Experimental Set Up}

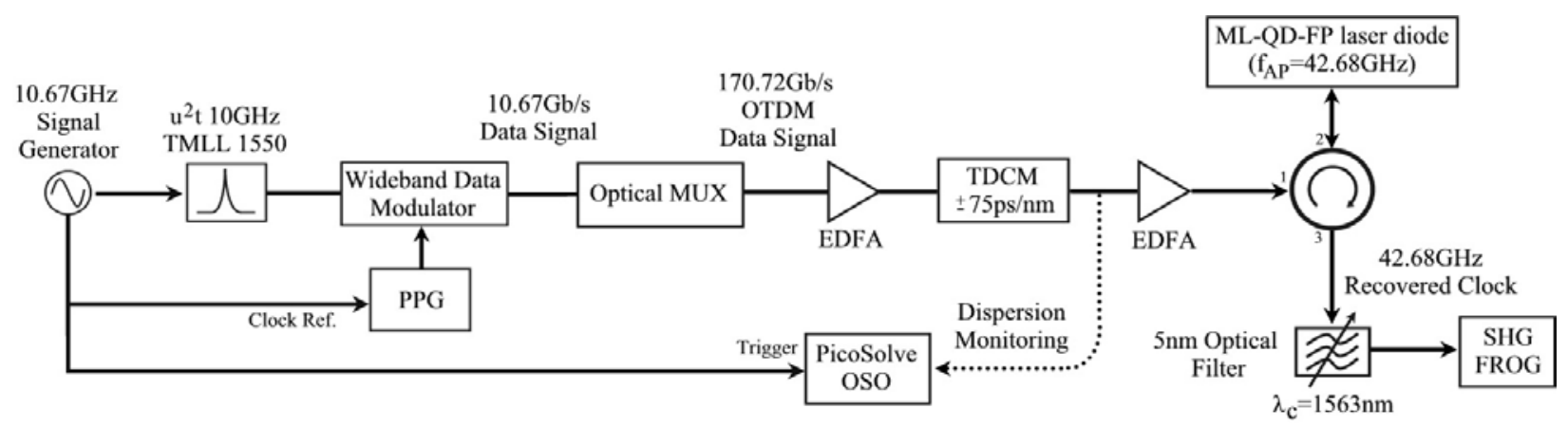

Fig.1: Experimental set up used for the characterization of the optical clock recovery (OCR) to variation of input dispersion

The experimental set up, available on the PERSYST Platform, is shown in Fig. 1. The 10.67GHz optical pulse train was provided by an actively mode-locked pulse source $\left(\mathrm{u}^{2} \mathrm{t}\right.$ TMLL) generating $2 \mathrm{ps}$ optical pulses at $1550 \mathrm{~nm}$. This signal was then modulated with a $2^{31}-1$ PRBS data signal generated using a programmable pattern generator (PPG) and wideband data modulator. The $10.67 \mathrm{~Gb} / \mathrm{s}$ data signal was multiplied up to $170.72 \mathrm{~Gb} / \mathrm{s}$ using a passive delay line optical multiplexer, and then amplified using an EDFA in order to overcome the insertion loss of the multiplexer. The signal then entered a tunable dispersion compensating module (TDCM, from TeraXion) which allowed the dispersion of the OTDM data signal to be varied between $\pm 75 \mathrm{ps} / \mathrm{nm}$. The effects of dispersion were monitored using 


\section{OWS4.pdf}

a 1 ps resolution Optical Sampling Oscilloscope (OSO, from PicoSolve Inc.). The dispersed signal than entered the OCR circuitry, which comprised of an optical circulator and the self-pulsating ML-QD-FP laser diode, which had a peak emission wavelength around 1570nm (L-band). By varying the bias current and temperature of the device, the FP laser's frequency was tuned close to $42.68 \mathrm{GHz}$ thereby allowing an optical clock to be extracted from the injected signal [7]. The optical power of the signal injected into the ML-QD-FP was in the region of 5-10dBm. The recovered clock signal was then optically filtered $(\mathrm{BW}=5 \mathrm{~nm}$, centred at $1563 \mathrm{~nm})$ to eliminate the input data signal. The temporal and phase profiles of the filtered signal was then analysed as a function of various dispersion levels using the SHG-FROG. The FROG measurement scheme is advantageous over other pulse measurement schemes such as bandwidth limited oscilloscopes and traditional autocorrelation methods as its retrieves intensity and corresponding phase information [9]. This property allows for the study of the dispersion transfer of the clock recovery process.

\section{Experimental Result}

Fig. 2 shows two eye diagrams as measured using the OSO of the high-speed OTDM data signal under two different dispersion regimes; (a) Zero dispersion; (c) $-75 \mathrm{ps} / \mathrm{nm}$ dispersion. The pulse separation in Fig. 2 (a) is 5.8ps corresponding to the multiplexed data rate, while the pulse width is approximately $3.2 \mathrm{ps}$. This pulse duration is slightly high for a $170 \mathrm{Gbit} / \mathrm{s}$ OTDM data signal and results from pulse broadening experienced in the EDFA before

(a)
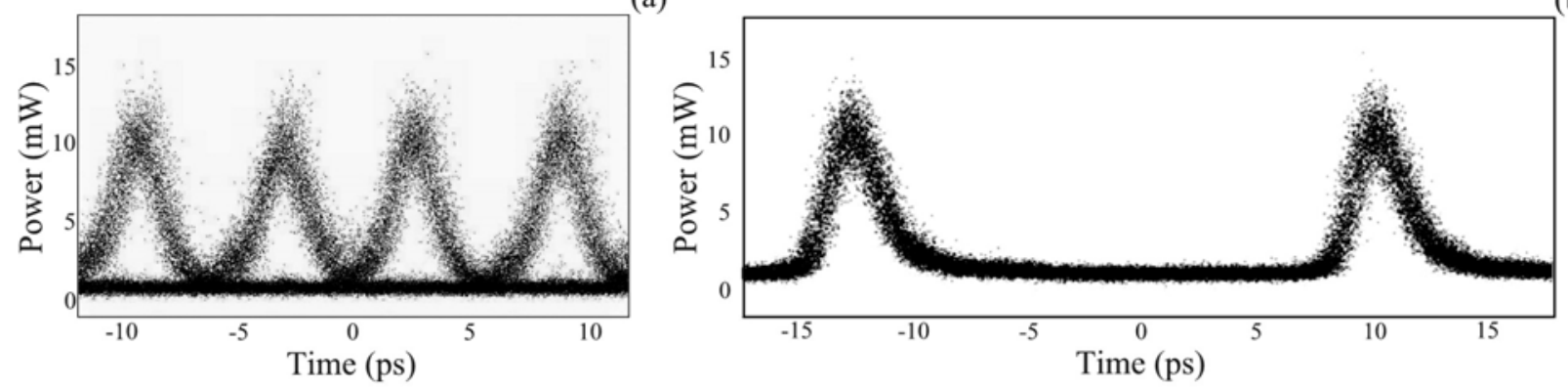

(b)

(c)
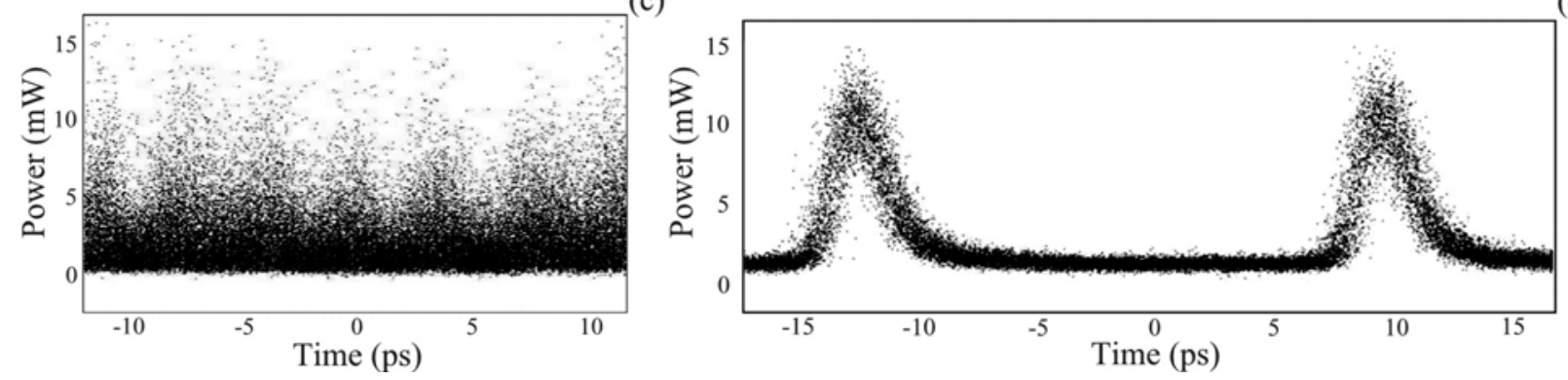

(d)

Fig.2: (a) Eye diagrams for 170Gb/s data signals for zero dispersion; (b) Recovered clock signal for zero dispersion; (c) Eye diagrams for170Gb/s data signals for $-75 \mathrm{ps} / \mathrm{nm}$ dispersion; (d) Recovered clock signal for $-75 \mathrm{ps} / \mathrm{nm}$ dispersion

the TDCM. Fig. 2 (c) displays the eye diagram for the same 170Gb/s OTDM data signal when the dispersion was set to $-75 \mathrm{ps} / \mathrm{nm}$. As expected the eye is completely closed due to adjacent pulses overlapping. Fig. 2 (b \& d) shows oscilloscope traces of the $42.68 \mathrm{GHz}$ optically recovered clock for the two different dispersion regimes. The pulse separation is $23.4 \mathrm{ps}$ which corresponds to a repetition rate of $42.68 \mathrm{GHz}$. The measured pulse duration on the OSO was in the order of $1.8 \mathrm{ps}$, while the temporal jitter was $<600 \mathrm{fs}$ for both signals. Fig. 3 (a) and (b) shows the retrieved pulse intensity and chirp profile respectively from the FROG measurement of the $42.68 \mathrm{GHz}$ recovered clock signals for two different dispersion regimes. Fig. 3 (a) shows that there is little difference in the temporal profile of the clock signal when the incident OTDM has no dispersion $(0 \mathrm{ps} / \mathrm{nm})$ and when the dispersion is set to $-75 \mathrm{ps} / \mathrm{nm}$. The measured pulse width was $1.5 \mathrm{ps}$, which is slightly less than that recorded using the OSO. This could be accounted for by the limited temporal resolution of the OSO. What is interesting is that the FROG analysis displays two pulse pedestals, positioned about $2.4 \mathrm{ps}$ away, and $15 \mathrm{~dB}$ down, from the main peak. These pedestals are not clearly visible on the oscilloscope trace. Fig. 3 (b) shows the recovered chirp profile for the two pulses shown in Fig. 2 (b) and (d). As shown, the recovered clock signal has a slight negative chirp across the central portion of the pulse. Fig. 3 (c) 


\section{OWS4.pdf}

plots the variation of the measured pulse width of the recovered clock signal as a function of varying input dispersion imparted on the data signal. There is only a slight variation (100fs) between the measured pulse duration of the clock signal across the entire dispersion range. Fig. 3 (d) presents a plot of the group delay measured across the pulse as a function of dispersion imparted by the TDCM on the input signal. As displayed, the group delay varies less than $1 \mathrm{ps} / \mathrm{nm}$ over the entire dispersion tuning range of $150 \mathrm{ps} / \mathrm{nm}$.

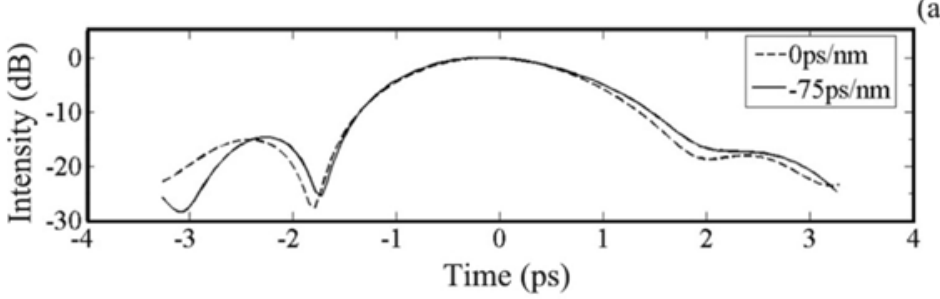

(a)

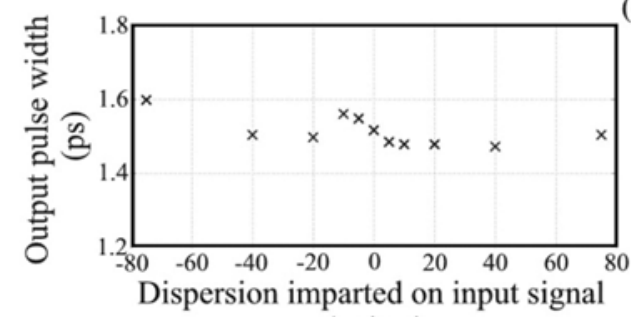

$(\mathrm{ps} / \mathrm{nm})$

(b)
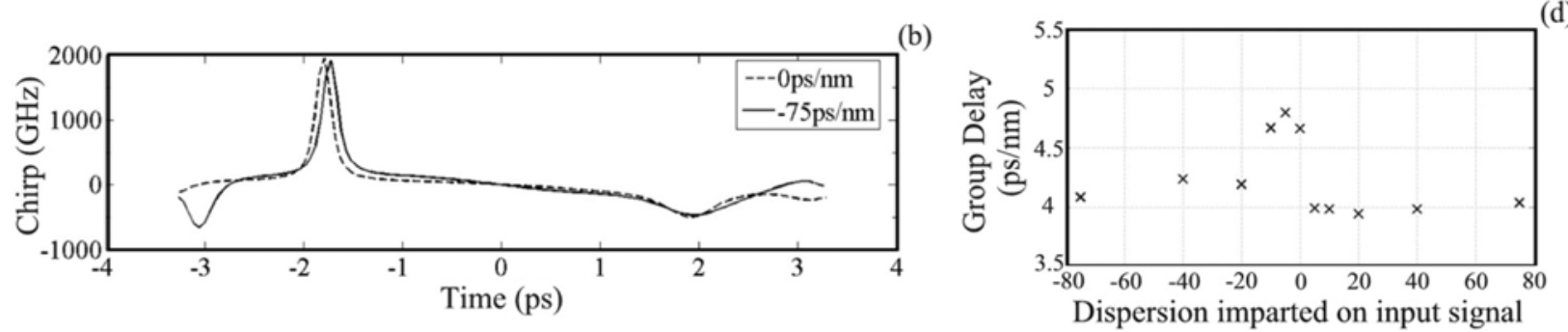

(ps/nm)

Fig.3: Retrieved FROG measurement of the (a) temporal profile and (b) chirp profile of the clock signal at $0 \mathrm{ps} / \mathrm{nm}$ and $-75 \mathrm{ps} / \mathrm{nm}$; (c) Plot of output pulse width versus input pulse dispersion; (d) Plot of clock signal group delay across the pulse as a function of dispersion imparted on the input signal.

\section{Conclusion}

This paper has presented an investigation into the effects of varying input dispersion levels has on the optical clock recovered signal based on ML-QD-FP laser diode. The effects of dispersion where monitored by using the SHGFROG methods, allowing direct analysis without the need for BER measurements. Results presented show that for a dispersion range of $150 \mathrm{ps} / \mathrm{nm}$, the group delay of the output signal varied by less than $1 \mathrm{ps} / \mathrm{nm}$. This demonstrates that the OCR technique presented is insensitive to distortions of the injected signal caused by chromatic dispersion. In addition, the temporal jitter on the recovered clock signal was $<600 \mathrm{fs}$ over the entire dispersion tuning range.

\section{References}

[1] A.D Ellis, K Smith, and D.M Patrick, “All optical clock recovery at bit rates up to $40 \mathrm{Gbit} / \mathrm{s”,} \mathrm{Electron.} \mathrm{Lett.} \mathrm{29,} \mathrm{1323-1324} \mathrm{(1993).}$

[2] R.Salem, A.A.Ahamdi, G.E.Tudury, G.M.Carter and T.E.Murphy, "Two-Photon Absorption for Optical Clock Recovery in OTDM Networks”, IEEE Light. Technol. 24, 3353-3362 ( 2006).

[3] O.Kamatani and S.Kawanishi, "Ultrahigh-Speed Clock Recovery with Phase Lock Loop Based in Four-Wave Mixing in a TravelingWave Laser Diode Amplifier”, IEEE Light. Technol. 14, 1757-1767 (1996).

[4] H. Yokoyama, Y. Hashimoto, H. Kurita, and I. Ogura, "All-optical subharmonic clock recovery and demultiplexing", Conference on Optical Fiber Communication (OFC) 3, 232-234 (2000).

[5] T.Ohno, K.Sato, T.Shimizu, T.Furuta and H.Ito, "Recovery of $40 \mathrm{GHz}$ optical clock from $160 \mathrm{Gbit} / \mathrm{s}$ data using regeneratively modelocked semiconductor laser", Electron. Lett. 39, 453-454 (2003).

[6] J.Renandier, B.Lavigne, M.Jourdan, P.Gallon, F.Lelarge, B.Dagens, A.Accard, O.Legouezigou and G-H.Duan, "First demonstration of all-optical clock recovery at $40 \mathrm{GHz}$ with standard-compliant jitter characteristics based on a quantum-dots self-pulsating semiconductor laser", European Conference and Exhibition on Optical Communications (ECOC) 6, 31-32 (2005).

[7] V. Roncin, A.O'Hare, S.Lobo, E.Jacquette, L.Bramerie, P.Rochard, W.-T.Le, M.Gay, J.-C.Simon, A.Shen, J.Renaudier, F.Lelarge and G.H.Duan, "Multi-Data-Rate System Performance of a 40-GHz All-Optical Clock Recovery Based on a Quantum-Dot Fabry-PÉrot Laser", IEEE Photon. Technol. Lett.19, 1409-1411 (2007).

[8] V.Roncin, S.Lobo, L.Bramerie, P.Rochard, A.Shen, F.Van Dijk, G.-H.Duan and J.C.Simon, "Demonstration of Chromatic Dispersion and Optical Noise insensitivity of a Quantum-Dash based \{F\}abry-Perot Laser in All-Optical Clock Recovery at 40Gbit/s", European Conference and Exhibition on Optical Communications (ECOC) 2, 165-166 (2007).

[9] R.Trebino, K.DeLong, D.N.Fittinghoff, J.N.Sweetser, M.A.Krumbugel and B.A.Richman, "Measuring ultrashort laser pulses in the timefrequency domain using frequency-resolved optical gating", Rev.Sci. Instrum, America Institute of Physics, 68,3277-3295 (1997). 